\title{
ERRATUM: "OBSERVATIONAL EVIDENCE OF TORUS INSTABILITY AS TRIGGER MECHANISM FOR CORONAL MASS EJECTIONS: THE 2011 AUGUST 4 FILAMENT ERUPTION” (2014, ApJ, 785, 88)
}

\author{
F. P. Zuccarello ${ }^{1,2}$, D. B. Seaton ${ }^{2}$, B. Filippov ${ }^{3}$, M. Mierla $^{2,4}$, S. Poedts $^{1}$, \\ L. A. Rachmeler ${ }^{2}$, P. Romano ${ }^{5}$, AND F. ZuCCARELlo ${ }^{6}$ \\ ${ }^{1}$ Centre for Mathematical Plasma-Astrophysics, KU Leuven, Celestijnenlaan 200B, B-3001 Leuven, Belgium; \\ Francesco.Zuccarello@wis.kuleuven.be, Stefaan.Poedts@wis.kuleuven.be \\ ${ }^{2}$ Royal Observatory of Belgium, Ringlaan 3, B-1180 Brussels, Belgium; dseaton@ oma.be, rachmeler@oma.be, marilena@oma.be \\ ${ }^{3}$ Pushkov Institute of Terrestrial Magnetism, Ionosphere and Radio Wave Propagation of the Russian Academy of Sciences \\ (IZMIRAN), Troitsk, Moscow 142190, Russia; bfilip@izmiran.ru \\ ${ }^{4}$ Institute of Geodynamics of the Romanian Academy Jean-Louis Calderon 19-21, RO-020032 Bucharest, Romania \\ 5 INAF-Osservatorio Astrofisico di Catania, via S. Sofia 78, I-95123 Catania, Italy; Paolo.Romano@ oact.inaf.it \\ ${ }^{6}$ Dipartimento di Fisica e Astronomia, Universitá di Catania, Via S. Sofia 78, I-95123 Catania, Italy; fzu@ oact.inaf.it \\ Received 2014 August 1; published 2014 October 28
}

Online-only material: color figures

In our recent paper (2014, ApJ, 785, 88), we reported the analysis of a filament eruption that occurred on 2011 August 4 in active region NOAA 11261. In our published paper, we discussed the morphology of the active region and its pre-eruptive dynamics. We used stereoscopic reconstructions to infer the three-dimensional location of the filament as well as potential magnetic field extrapolations to determine the decay index at the location of the filament. The analysis strongly suggested that the torus instability was the trigger of the eruption. Since publication, we found (1) an oversight in the protocol that we used to identify regions of magnetic flux to
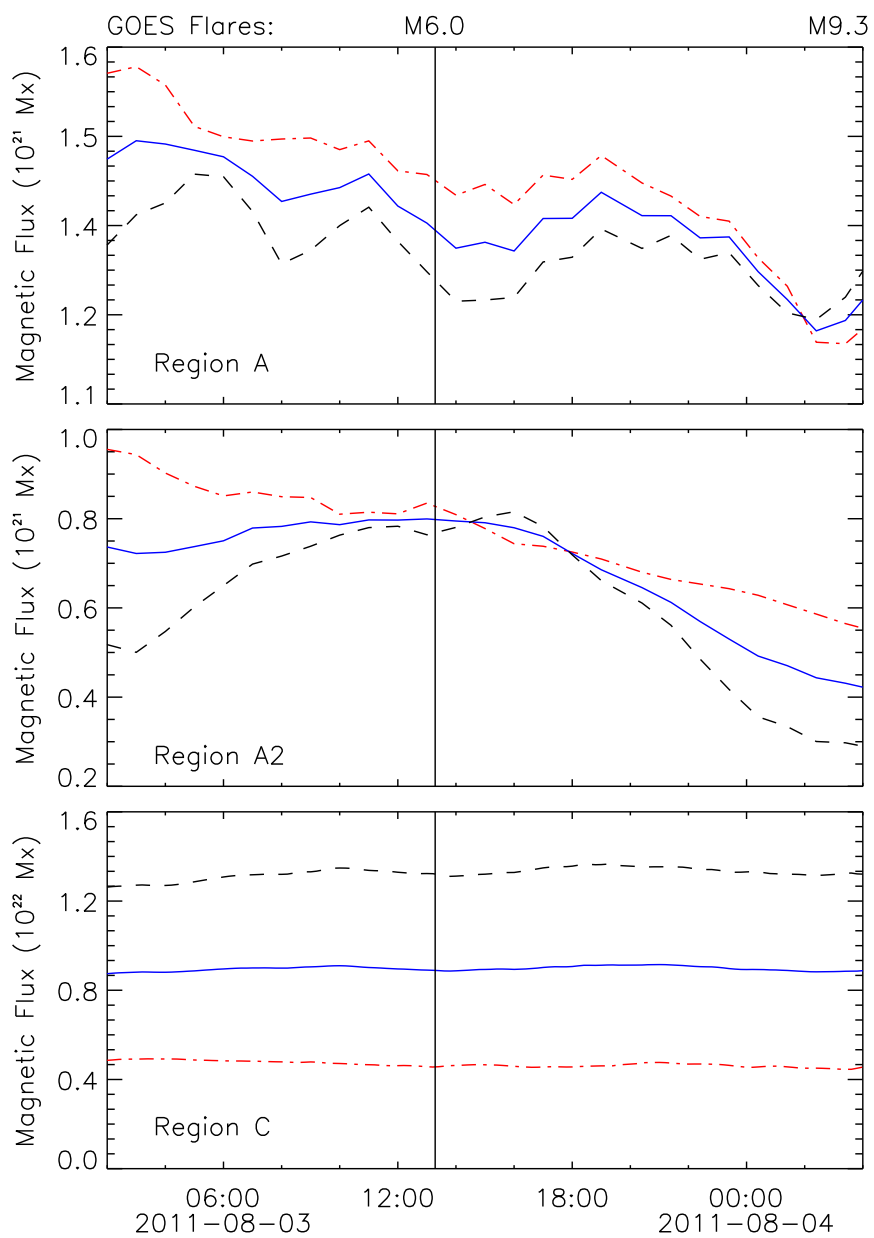

Figure 5. Time evolution of the positive (dash-dotted red), negative (dashed black), and total unsigned (solid blue) magnetic flux for Region A (top), Region A2 (middle), and Region C (bottom).

(A color version of this figure is available in the online journal.) 


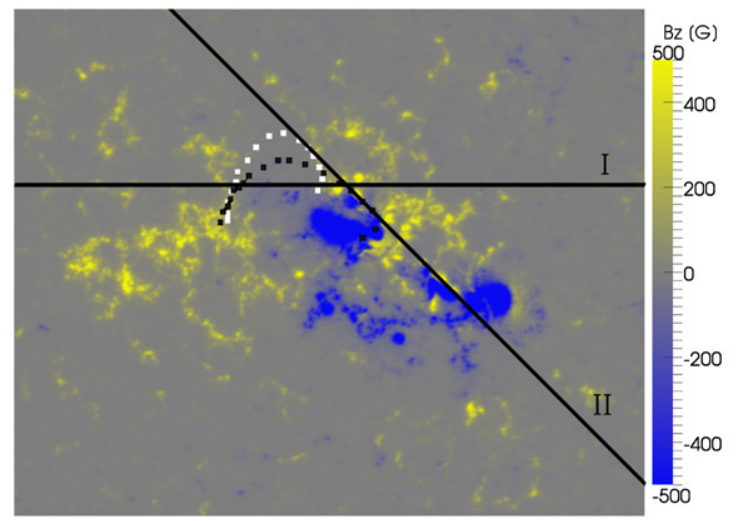

(a)

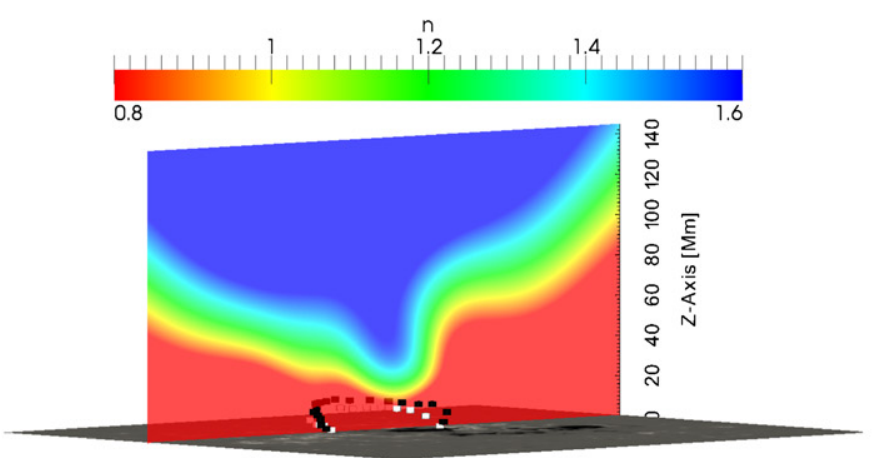

(c)

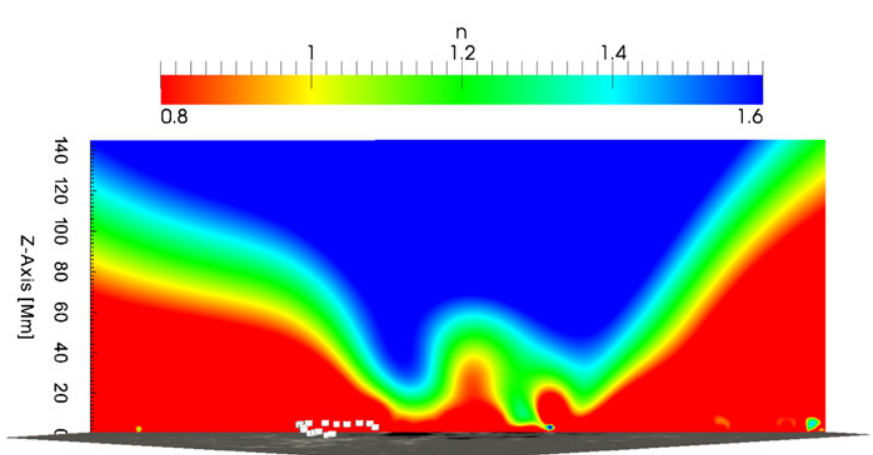

(b)

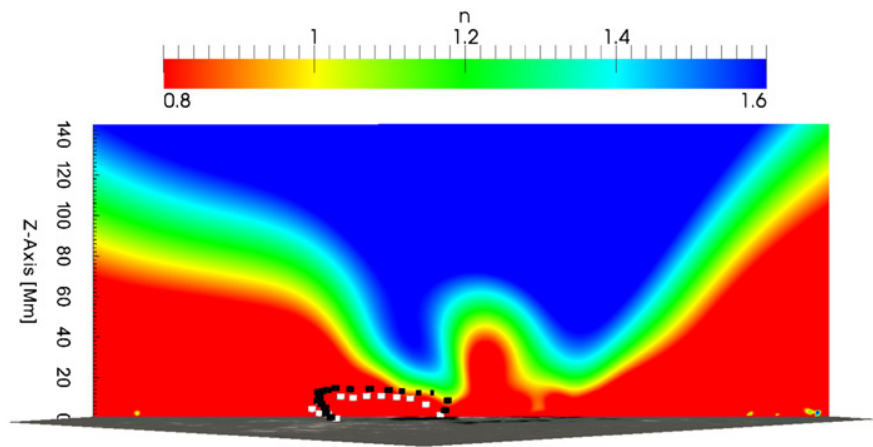

(d)

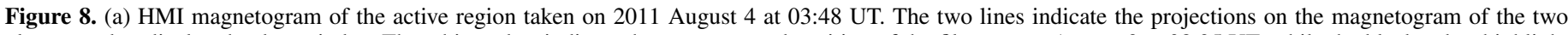

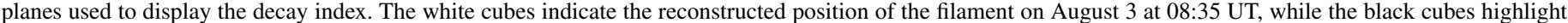

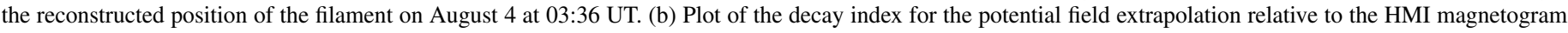

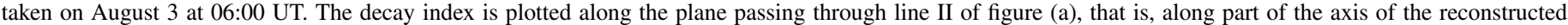

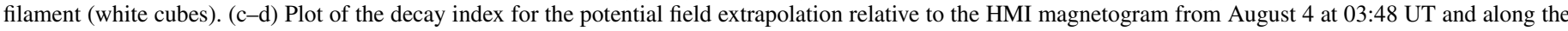

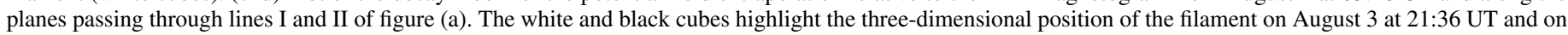
August 4 at 03:36 UT, respectively.

(A color version of this figure is available in the online journal.)

be tracked using the YAFTA algorithm and (2) a factor of two underestimation in the conversion from pixel size to physical units. Although these oversights do not influence the analysis or the overall conclusion of the paper, they do affect some of the figures and the value of the decay index for any given height. We regret that these errors were not discovered before publication and here we describe the resultant changes and provide updated figures where necessary.

In our published paper, we used a sequence of co-aligned Helioseismic and Magnetic Imager (HMI) magnetograms to compute the evolution of the magnetic flux. As a consequence of the underestimation of the pixel size, the magnetic flux was underestimated by a factor of four. Furthermore, the protocol used to identify regions of magnetic flux to be tracked by YAFTA originally only considered those regions that were tangent to the polarity inversion line at step zero, but regions that emerged at the polarity inversion line should also have been included. This led to the omission of one small area of flux from the analysis. Although this omission results in a change in the fine structure of the plot of Region A, the overall trend is unaffected. Figure 5 of this erratum updates that of our published paper.

In our paper, we used the DAVE algorithm to derive the velocity maps for AR 11261. As a consequence of the underestimation of the conversion factor, the FWHM of the resolving window was 10 arcsec and not 5 arcsec as originally stated in our published paper and the velocity flows were underestimated by a factor two. Therefore, in Figure 6 , the arrow scale is not $0.3 \mathrm{~km} \mathrm{~s}^{-1}$, but $0.6 \mathrm{~km} \mathrm{~s}^{-1}$.

The error in the conversion factor also affected Figures 8 and 9. Updated versions of these figures are included in this erratum. In particular, Figure 8 shows the computed decay index along the two selected planes for the magnetic field extrapolations performed using the magnetograms obtained on August 3 at 06:00 UT and on August 4 at 03:48 UT. Figure 8(b) shows that before the M6 flare, the filament is located in a region that is stable with respect to the torus instability. However, after the M6 flare (Figures 8(c) and (d)), the filament approaches the region where the decay index is about 1-1.1. This is the main difference from the result presented in our published paper, where the yellow-green region indicated a value of the decay index of about 1.3-1.5.

We would like to note that this result does not affect the overall conclusion of our published paper. In fact, the critical decay index for straight flux ropes is unity in the classical treatments of van Tend \& Kuperus (1978) and Molodenskii \& Filippov (1987). However, the more recent treatment by Démoulin \& Aulanier (2010) yields a small positive correction, so that a value above unity should be expected. These authors showed that a plausible range for the critical value of the decay index for expanding solar flux ropes is about 1.1-1.3. We now find that at the moment of the eruption the filament was located at a height where the decay index is $n \gtrsim 1$, that 

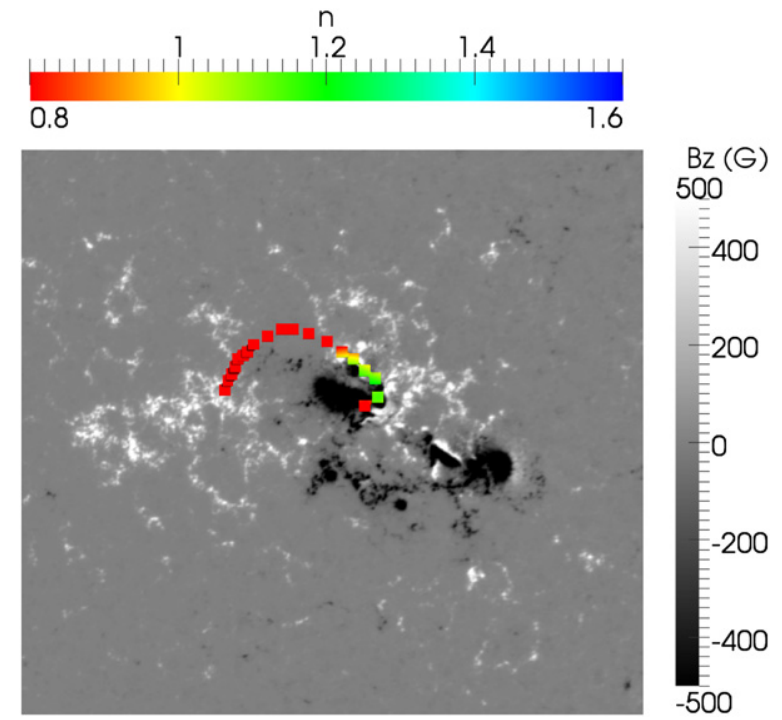

Figure 9. HMI magnetogram of the active region taken on 2011 August 4 at 03:48 UT together with the reconstructed position of the filament on August 4 at 03:36 UT The reconstructed filament is color-coded with the decay index computed from the potential field extrapolation. North is at the top and west to the right.

(A color version of this figure is available in the online journal.)

is, very close to the lower limit of the instability interval proposed by Démoulin \& Aulanier (2010), especially if we notice that the axis of the assumed flux rope can actually lie above the top of the visible filament and hence the inferred values of the critical decay index are lower limits of the actual critical decay index. The updated result also appears to be consistent with the geometry of the filament. In fact, as shown in Figure 8, the filament does not seem to have a toroidal shape such that the separation of the foot points is more than two times the height of the filament. For such non-toroidal flux ropes, the critical index would be close to the lower limit identified by Démoulin \& Aulanier (2010), since this limit corresponds to straight line currents instead of toroidal line currents.

In conclusion, the value of the decay index near the filament at the moment of the eruption has changed and is now $n \gtrsim 1$. This is consistent with the critical value of the onset of the instability for flux ropes with nearly translational invariant geometry.

\section{REFERENCES}

Démoulin, P., \& Aulanier, G. 2010, ApJ, 718, 1388

Molodenskii, M. M., \& Filippov, B. P. 1987, AZh, 64, 1079

van Tend, W., \& Kuperus, M. 1978, SoPh, 59, 115 